OPEN ACCESS

Edited by:

Ruth Heying,

University Hospital Leuven, Belgium

Reviewed by:

Hala El-Bassyouni,

National Research Centre, Egypt

Emanuele Monda

University of Campania Luigi

Vanvitelli, Italy

*Correspondence:

Hae Soon Kim

hyesk@ewha.ac.kr

Specialty section: This article was submitted to

Pediatric Cardiology,

a section of the journal

Frontiers in Pediatrics

Received: 22 July 2021 Accepted: 22 September 2021

Published: 22 October 2021

Citation:

Lee JW, Hong YM and Kim HS (2021) Identification of Cardiovascular Risk Factors in Obese Adolescents With Metabolic Syndrome.

Front. Pediatr. 9:745805.

doi: 10.3389/fped.2021.745805

\section{Identification of Cardiovascular Risk Factors in Obese Adolescents With Metabolic Syndrome}

\author{
Jung Won Lee, Young Mi Hong and Hae Soon Kim* \\ Department of Pediatrics, Ewha Womans University School of Medicine, Seoul, South Korea
}

Objective: There are studies that show different associations between metabolic syndrome (MS) and cardiovascular disease in adolescent. This study is aimed to identify probable cardio-vascular risk factors in obese adolescents with MS.

Methods: Sixty-five obese adolescents with a body mass index (BMI) > 95 percentile were enrolled and divided into two groups with MS or without MS. Left ventricular mass (LVM), left ventricular mass index, ejection fraction, epicardial fat thickness, visceral fat thickness (VFT) and carotid intima-media thickness were measured. Anthropometric and blood chemistry parameters were estimated. Above parameters were compared based on presence or absence of MS.

Results: The prevalence of MS was $23.1 \%$ in obese adolescents. LVM showed significant correlation with body mass index (BMI), hip circumference $(\mathrm{HC})$, fat mass, total cholesterol (TC), LDL-cholesterol (LDL-C) and waist circumference (WC). VFT significantly correlated with WC, BMI, hip circumflex $(\mathrm{HC})$, obesity index $(\mathrm{Ol})$, fat \%, fat mass, insulin, TC, LDL-C, insulin, triglyceride (TG), glucose, homeostatic model assessment for insulin resistance (HOMA-IR) and leptin.

Conclusions: Screening for the MS in overweight adolescents may help to predict risk of future cardiovascular disease. These data suggest that LVMI and VFT are significant parameters for predicting cardiovascular disease risk in obese adolescents.

Keywords: metabolic syndrome, visceral obesity, left ventricular mass index, visceral fat thickness, cardiovascular disease

\section{INTRODUCTION}

The prevalence of adolescent metabolic syndrome (MS) has increased substantially due to lack of physical activity, unhealthy diet and lifestyle, and incomplete sleep due to presence of electrical devices.

However, there was no definition of adolescent MS until now, and most definitions have been adapted from the Adult Treatment Panel III (ATP III) definition, published by Cook et al. (1). The International Diabetes Federation (IDF) released its unique definition of MS in children and adolescents in 2007 (i.e., IDF 2007 definition), at the age of $10 \mathrm{yr}$ and more, MS requires the presence of abdominal obesity ( $\geq 90$ th percentile) plus, the presence of two or more of elevated triglycerides (TG) $(\geq 150 \mathrm{mg} / \mathrm{dL})$, low high-density lipo-protein-cholesterol (HDL-C) $(<40 \mathrm{mg} / \mathrm{dL}$ ), high blood pressure (Systolic blood pressure $\geq 130$ or diastolic blood pressure $\geq$ $85 \mathrm{~mm} \mathrm{Hg}$ ), and elevated plasma glucose (Fasting glucose $\geq 100 \mathrm{mg} / \mathrm{dL}$ or known type2 DM) (2). 
Visceral fat and insulin resistance are key factors of MS development (3). The visceral fat accumulation is associated with vascular atherosclerosis and cardiovascular outcome after all (4). These consequences are from pro-inflammatory cytokines originated from visceral fat tissue (5). For example, epicardial fat around heart interacts with myocardium and coronary arteries through cytokines (6). Thus, definition of MS has expanded to include many inflammatory cytokines (7). It has been postulated that obesity dysregulates blood adipokine levels, may induce insulin resistance, and finally influence the development of MS and vascular complications (8).

Although increased number of metabolic risk factors and their complex interactions contribute to the occurrence of MS, this has not been well-studied in adolescent populations (9).

The aim of this study was to identify specific risk factors from visceral obesity closely related to future cardiovascular consequence in obese adolescent populations.

\section{METHODS}

\section{Study Participants}

Sixty five obese adolescents ( 31 males, 34 females) aged between 15 and 17 in a high school participated in this study. They were divided into two groups: the MS group (total $=15$, male $=9$, female $=6$ ) and the group without MS (total $=50$, male $=22$, female $=28$ ). We measured MS in adolescents using a modified cook's definition. Adolescent obesity was defined as body mass index (BMI) above the 95th percentile for age and sex by the survey of Korean Centers of Disease Control and Prevention in 2007 (10). Adolescents with other endocrine disease, psychiatric problem or other severe and chronic disease were excluded. Informed consents were obtained from the parents or guardians. The study was conducted according to the guidelines of the Declaration of Helsinki, and approved by Ethical Committee of Ewha Womans University, College of Medicine, Seoul, Korea (approval No: 0319/12.06.2020).

\section{Anthropometric Measurement}

Anthropometric data of weight, height, waist circumference (WC), BMI, obesity index (OI), hip circumflex (HC), fat mass, fat percentage and blood pressure (BP) from both groups were obtained. Both the height and weight were taken with light dressed and shoes were off on the height measuring instrument (JENIX, Samhwa Co., Ltd., Seoul, Korea), and the eyes were straightened. The vertical distance from the soles of the feet to the top of the head was measured and recorded up to $0.1 \mathrm{~cm}$. The weight was recorded up to $0.1 \mathrm{~kg}$. WC was measured at the mid-point between 12th rib and the mid portion of the superior iliac crest. BMI was calculated by dividing the body weight (kilogram) by height (meter) in meter square. OI was obtained by the equation using the standard weight value corresponding to the 50th percentile of the weight data chart for Korean children. $\mathrm{HC}$ was measured from the most protruding part of the hip. Fat mass and fat percentage were measured by bioelectric impedance analysis (BIA) (InBody 720, Biospace Co., Ltd., Seoul, Korea). BIA can be used as an alternative to gold standard dual-energy Xray absorptiometry (DXA) admitting BIA underestimate body fat percentage values compared to DXA (11). BP was measured after 10 min of bed rest using an automatic oscillometric BP device in a supine position (Omron HEM-7320-LA, Omron Healthcare Co. Ltd, Kyoto, Japan).

\section{Laboratory Measurement}

Blood was taken from all 65 subjects after $14 \mathrm{~h}$ fasting. Blood glucose, total cholesterol, TG, low density lipoproteincholesterol (LDL-C), HDL-C, aspartate aminotransferase (AST), alanine aminotransferase (ALT), Serum TNF- $\alpha$ and IL-6 levels were measured by sandwich enzyme immunoassay-based Quantikine Human interleukine and TNF- $\alpha$ kit (R\&D system Inc., Minneapolis, MN, USA). Serum leptin, adiponectin and insulin levels were measured using the Human Leptin 125 tubes radioimmunoassay kit (Linco Resarch Inc., St. Charles, MO, USA), radioimmunoassay human adiponectin 125 tubes radioimmunoas-say kit (Linco Research, Inc. St. Charles, MO, USA) and human insulin chemiluminescence immunoassay kit (insulin, Siemens Centaur, Holliston, MA, USA), respectively.

Insulin resistance was measured by the homeostasis model assessment of insulin resistance (HOMA-IR), by dividing the multiple of insulin $(\mu \mathrm{U} / \mathrm{mL})$ and serum glucose $(\mathrm{mmol} / \mathrm{L})$.

\section{Carotid Artery Ultrasound Measurement}

Carotid intima-media thickness (CIMT) was measured with neck ultrasound iU22 (Intelligent Ultrasound System, Philips Medical System, Amsterdam, The Netherlands). After the subjects lied down and rest for at least $30 \mathrm{~min}$, probe was placed on common carotid artery $1 \mathrm{~cm}$ proximal from the carotid bifurcation. Three points of thickest intima-media lengths were measured and average was recorded.

\section{Abdominal Ultrasonography Measurement}

Abdominal fat thickness including subcutaneous fat thickness, visceral fat thickness and pre-peritoneal fat thickness were measured with abdominal ultrasound Acuson XP128 (Acuson, Mountain View, CA, USA) by placing a $3.5 \mathrm{MHz}$ lineararray probe $1 \mathrm{~cm}$ above the navel at end-expiratory phase. Subcutaneous fat thickness was measured from skin to anterior abdominal muscle, visceral fat thickness from posterior abdominal muscle to anterior wall of aorta, and pre-peritoneal fat thickness from the subcutaneous layer to the peritoneum, respectively. Same experienced radiologist performed the measurement.

\section{Echocardiographic Parameters Measurement}

Echocardiographic parameters such as epicardial fat thickness (EFT), LV dimension, and systolic/diastolic parameters were estimated by echocardiography (Acuson Sequoia-C 512, Siemens, CA, USA). The subjects lied down in left lateral decubitus with using phased-array echocardiograms in M-mode, $2 \mathrm{D}$, and pulsed and color-flow Doppler settings. EFT on the free wall of right ventricle was measured in parasternal long axis and short axis views. LV dimension was measured by perpendicular to the long axis of the left ventricle. Systolic and diastolic parameters such as stroke volume (SV) was determined by 
the aortic annular cross-sectional area multiplied by the aortic time-velocity integral. Cardiac output (CO) was calculated by multiplying SV and the heart rate (HR). Ejection fraction (EF) was calculated from biplane Simpson formula and fractional shortening (FS) was calculated using left ventricular internal dimensions. Early diastolic (E), late atrial (A) peak velocities, $\mathrm{E} / \mathrm{A}$ ratios, and E-wave deceleration time ( $\mathrm{ms}$ ) were measured from pulsed doppler mode in apical window. Left ventricular mass (LVM) was calculated calculated from American Society of Echocardiography guideline, and left ventricular mass index (LVMI) was calculated from LMV devided by body surface area (Monstella method).

$\operatorname{LVM}(\mathrm{g})=0.8[1.04($ STd + LVIDd + PWTd $) 3-$ LVIDd3 $]+$ $0.6 \mathrm{~g}$

$\square$ STd = interventricular septal thickness at end-diastole ( $\mathrm{mm})$

$\square$ LVIDd $=$ LV end-diastolic dimension $(\mathrm{mm})$

$\square$ PWTd $=$ posterior wall thickness at end-diastole $(\mathrm{mm})$

Body surface area: $\{[\mathrm{Ht}(\mathrm{cm}) \times \text { Bwt }(\mathrm{kg})] / 3,600\}^{1 / 2}$

Ht: Height Bwt; body weight.

\section{Brachial-Ankle Pulse Wave Velocity and Ankle Brachial Index Measurement}

Brachial-ankle pulse wave velocity (baPWV) were measured using a volume-plethysmographic apparatus (Colin Co. Ltd., Komaki, Japan). The subject was examined in supine position for at least $5 \mathrm{~min}$ with taking their usual medication. The device gathered oscillometric waveforms and simultaneously calculates the time intervals between brachium and ankle waveforms. Two sets of baPWV values were measured and left and right data were showed. Ankle Brachial index (ABI) was calculated as the ratio of systolic BP in ipsilateral ankle and brachium.

\section{Statistical Analysis}

We performed statistical analyses using Statistical Package for the Social Sciences (SPSS) (version 20, SPSS Inc. Chicago, IL, USA). Data were presented as means and standard deviations. The comparison of continuous variables was done using the Student $t$-test or two-way ANOVA. Univariate and multivariate logistic regression models were implemented to examine the independent relationship between the dependent variable MS and other variables of metabolic parameters. $P$-value $<0.05$ was considered as statistically significant.

\section{RESULTS}

\section{The Prevalence of Metabolic Syndrome and Clustering of Metabolic Components}

Of the total of sixty-five subjects ( 31 males, 34 females), $23.1 \%$ $(15 / 65)$ showed an incidence of MS that satisfied more than three of the criteria of MS.

The percentage of the subjects meeting MS criteria were WC $\geq$ 90 percentile $(52.3 \%)$, systolic $\mathrm{BP}$ or diastolic $\mathrm{BP} \geq 90$ percentile (mmHg) (52.3\%), and HDL- C $\leq 40$ (mg/dL) (46.2\%), followed by $\mathrm{TG} \geq 110(\mathrm{mg} / \mathrm{dL})(26.2 \%)$ and fasting glucose $\geq 100(\mathrm{mg} / \mathrm{dL})$ (3.1\%) in Table 1.
TABLE 1 | Prevalence of components of MS in obese adolescents.

\begin{tabular}{lcc}
\hline Criteria & $\boldsymbol{N}(\mathbf{M} / \mathbf{F})$ & $\%$ \\
\hline WC $\geq 90$ percentile & $34(17 / 17)$ & 52.3 \\
SBP or DBP $\geq 90$ percentile $(\mathrm{mmHg})$ & $34(13 / 21)$ & 52.3 \\
HDL-C $<40(\mathrm{mg} / \mathrm{dL})$ & $30(17 / 13)$ & 46.2 \\
Triglyceride $\geq 110(\mathrm{mg} / \mathrm{dL})$ & $17(11 / 6)$ & 26.2 \\
Fasting glucose $\geq 100(\mathrm{mg} / \mathrm{dL})$ & $2(1 / 1)$ & 3.1 \\
\hline
\end{tabular}

MS, metabolic syndrome; WC, waist circumference; SBP, systolic blood pressure; $D B P$, diastolic blood pressure; HDL-C, high density lipotrotein-cholesterol; N, number; $\%$, percent from total subjects.

\section{Comparison of Anthropometric Data}

There was no difference in anthropometric data between MS and non-MS groups of both females and males in Table 2.

\section{Comparison of Biochemistry Parameters}

LDL-C was significantly increased in MS females (131.1 \pm 19.5 vs. $96.7 \pm 27.5 \mathrm{mg} / \mathrm{dL}, p=0.007)$, TG was significantly increased and HDL-C was significantly decreased in both MS males and MS females than control group. adolescents without MS. Adiponectin and insulin like growth factor binding protein 3 (IGFBP-3) were significantly decreased in MS females compared to non-MS females in Table 3.

\section{Comparison of the Geometrical Analysis of the Carotid Artery}

The geometrical analysis of the carotid artery had no differential data including intimal media thickness between two groups of both females and males in Table 4 .

\section{Comparison of Abdominal Sonographic Data}

In the abdominal sonographic data, VFT was significantly higher in MS females compared to non-MS females (42.0 \pm 7.5 vs. 31.1 $\pm 6.9 \mathrm{~mm}, p=0.004)$ in Table 5 .

\section{Comparison of Echocardiographic Data}

In the echocardiography data including epicardial fat thickness, there was significant increase in left ventricular mass (LVM) $(205.9 \pm 44.5$ vs. $258.7 \pm 59.0 \mathrm{~g}, p=0.008)$ and left ventricular mass index (LVMI) $\left(1.9 \pm 0.4\right.$ vs. $\left.2.4 \pm 0.5 \mathrm{~g} / \mathrm{m}^{2}, p=0.047\right)$ in MS (+) males in Table 6.

\section{Comparison of Pulse Wave Velocity and Ankle Brachial Index}

There was significant increase of left ABI in the MS (+) females $(106 \pm 6.8$ vs. $100.4 \pm 7.9, p=0.021)$. However, no significant difference was observed in heart rate, RbaPWV, LbaPWV and right ABI between the MS (-) and MS (+) groups in Table 7.

\section{Correlation Between the LV Mass and Other Metabolic Parameters}

LV mass is significantly associated with BMI, HC, fat mass, insulin, TC, LDL-C, and WC $(P<0.05)$ in Table 8. 
TABLE 2 | Comparison of anthropometric data in obese adolescents.

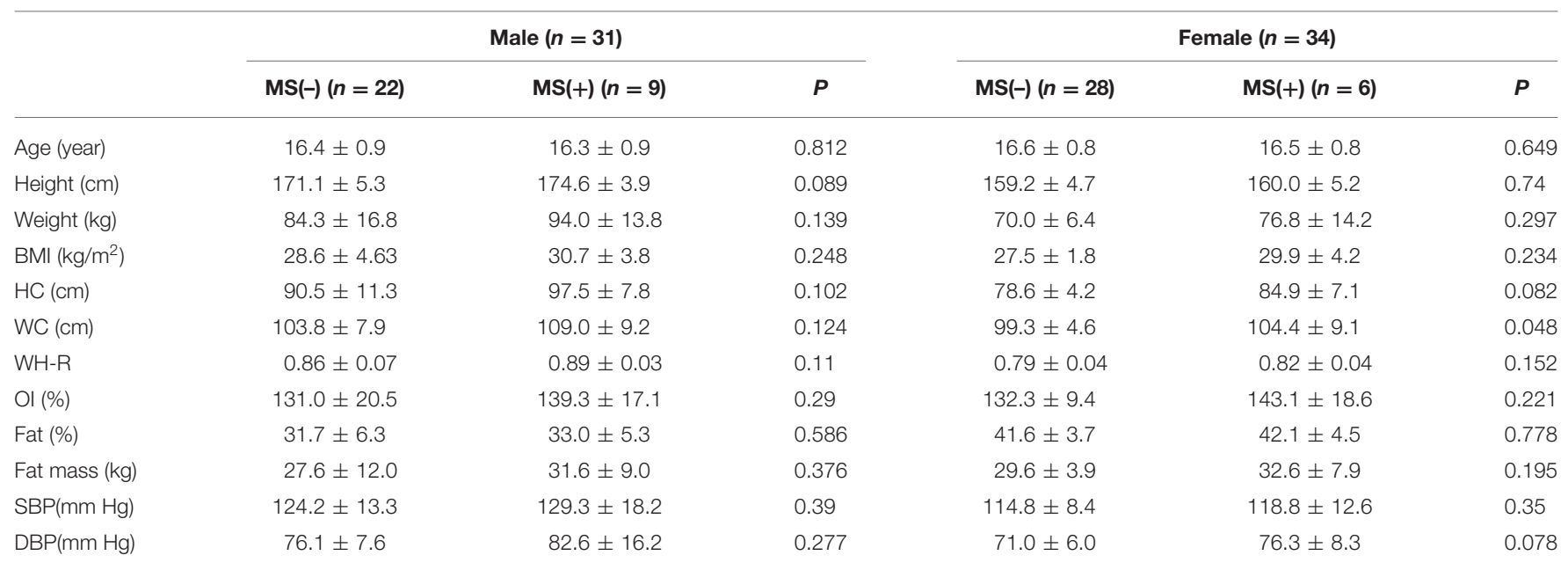

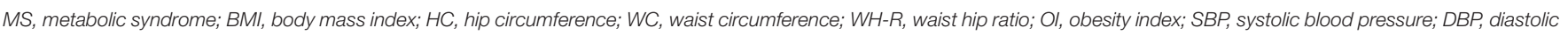
blood pressure.

TABLE 3 | Comparison of biochemistry parameters in obese adolescents.

\begin{tabular}{|c|c|c|c|c|c|c|}
\hline & \multicolumn{3}{|c|}{ Male $(n=31)$} & \multicolumn{3}{|c|}{ Female $(n=34)$} \\
\hline Insulin (mU/L) & $17.1 \pm 9.1$ & $18.9 \pm 10.4$ & 0.667 & $13.0 \pm 5.7$ & $24.1 \pm 21.5$ & 0.265 \\
\hline HOMA-IR & $3.8 \pm 1.8$ & $4.0 \pm 2.3$ & 0.742 & $2.7 \pm 1.2$ & $5.6 \pm 5.8$ & 0.277 \\
\hline TC (mg/dL) & $161.7 \pm 33.8$ & $152.7 \pm 36.5$ & 0.517 & $165.8 \pm 31.1$ & $196.6 \pm 24.3$ & 0.031 \\
\hline $\mathrm{TG}(\mathrm{mg} / \mathrm{dL})$ & $81.4 \pm 36.4$ & $145.3 \pm 60.9$ & 0.001 & $61.6 \pm 26.8$ & $151.8 \pm 36.1$ & 0.001 \\
\hline $\mathrm{HDL}-\mathrm{C}(\mathrm{mg} / \mathrm{dL})$ & $51.0 \pm 6.8$ & $45.5 \pm 2.6$ & 0.003 & $59.4 \pm 9.8$ & $44.6 \pm 2.6$ & 0.000 \\
\hline AST (IU/L) & $22.2 \pm 6.8$ & $39.1 \pm 22.5$ & 0.056 & $22.0 \pm 6.4$ & $30.3 \pm 30.2$ & 0.533 \\
\hline ALT (IU/L) & $29.9 \pm 16.0$ & $52.7 \pm 34.2$ & 0.086 & $17.5 \pm 10.1$ & $47.5 \pm 65.1$ & 0.313 \\
\hline hs-CRP (mg/L) & $1.4 \pm 2.1$ & $1.2 \pm 2.0$ & 0.828 & $1.6 \pm 2.0$ & $1.5 \pm 1.3$ & 0.888 \\
\hline IL-6 (pg/mL) & $6.5 \pm 3.3$ & $4.9 \pm 1.7$ & 0.21 & $7.1 \pm 7.1$ & $6.2 \pm 1.4$ & 0.782 \\
\hline FFA (uEq/L) & $561.6 \pm 294.5$ & $660.8 \pm 358.4$ & 0.434 & $655.3 \pm 429.4$ & $777.1 \pm 174.3$ & 0.504 \\
\hline IGFBP-3 (ng/mL) & $3782.7 \pm 508.7$ & $3577.3 \pm 413.4$ & 0.295 & $3598.7 \pm 491.3$ & $4108.3 \pm 258.4$ & 0.02 \\
\hline
\end{tabular}

MS, metabolic syndrome; HOMA-IR, homeostasis model assessment of insulin resistance; AST, aspartate aminotransferase; ALT, alanine aminotransferase; TC, total cholesterol; LDL-C,

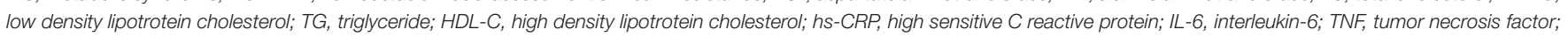
FFA, free fatty acid; IGFBP, insulin growth factor binding protein.

\section{Correlation Between Visceral Fat Tissue and Other Metabolic Parameters}

Visceral fat tissue is significantly associated with WC, DBP, TG, LDL-C, TC, glucose, BMI, HC, waist-hip ratio (WHR), OI, Fat mass, insulin, HOMA-IR, and leptin in Table 9.

\section{DISCUSSION}

Given that there is no well-established uniform definition of childhood MS (1), most frequently used definitions have been adopted from the Adult Treatment Panel III (ATP III) definition, modified and adapted by Cook et al. (12). And the IDF made the definition of MS in children $\geq 10$ years of age in 2007 to find the global prevalence of MS. These two measurement definitions had different cut off levels for hyperglyceridemia and elevated blood pressure. In this study of MS in obese adolescents, we applied modified Cook's definitions of the ATP III definitions.

Prevalence of MS in adolescent in representative studies was $28.7 \%$ in the study of cook et al. (12). In this study, we found the 
TABLE 4 | Comparison of geometrical parameters of carotid artery in the obese adolescents.

\begin{tabular}{|c|c|c|c|c|c|c|}
\hline & \multicolumn{3}{|c|}{ Male $(n=31)$} & \multicolumn{3}{|c|}{ Female $(n=34)$} \\
\hline & $\mathrm{MS}(-)(n=22)$ & $\operatorname{MS}(+)(n=9)$ & $P$ & $\operatorname{MS}(-)(n=28)$ & $\operatorname{MS}(+)(n=6)$ & $P$ \\
\hline IMT (mm) & $0.6 \pm 0.2$ & $0.6 \pm 0.1$ & 0.67 & $0.5 \pm 0.1$ & $0.6 \pm 0.2$ & 0.506 \\
\hline S diameter (mm) & $6.4 \pm 0.1$ & $6.4 \pm 0.7$ & 0.827 & $5.3 \pm 0.5$ & $5.3 \pm 0.5$ & 0.997 \\
\hline D diameter (mm) & $6.5 \pm 0.7$ & $13.8 \pm 21.5$ & 0.367 & $6.0 \pm 0.5$ & $17.2 \pm 24.5$ & 0.366 \\
\hline $\operatorname{LCSA}\left(\mathrm{m}^{2}\right)$ & $28.7 \pm 7.0$ & $28.8 \pm 4.2$ & 0.986 & $22.3 \pm 4.0$ & $22.5 \pm 4.1$ & 0.895 \\
\hline WCSA $\left(m^{2}\right)$ & $12.4 \pm 6.2$ & $11.5 \pm 3.3$ & 0.689 & $9.3 \pm 2.5$ & $10.9 \pm 4.8$ & 0.284 \\
\hline CSC & $0.2 \pm 0.1$ & $0.1 \pm 0.1$ & 0.207 & $0.1 \pm 0.1$ & $0.2 \pm 0.1$ & 0.179 \\
\hline CSD & $0.01 \pm 0.00$ & $0.00 \pm 0.00$ & 0.183 & $0.01 \pm 0.00$ & $0.01 \pm 0.00$ & 0.208 \\
\hline
\end{tabular}

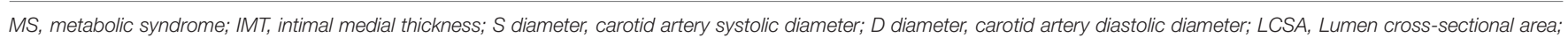
WCSA, wall cross-sectional area; CSC, cross-sectional compliance; CSD, cross-sectional distensibility.

TABLE 5 | Comparison of abdominal sonographic data in the obese adolescents.

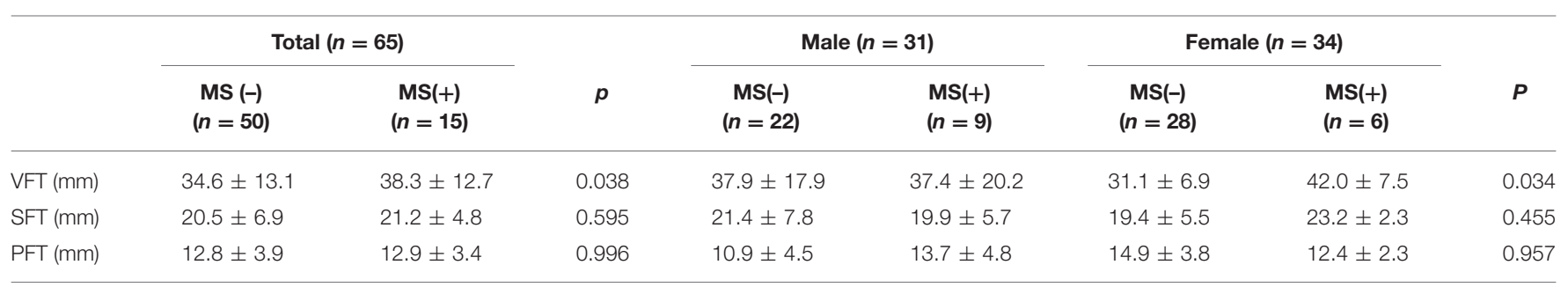

VFT, visceral fat tissue; SFT, subcutaneous fat tissue; PFT, properitoneal fat tissue.

TABLE 6 | Comparison of echocardiographic data in the obese adolescents.

\begin{tabular}{|c|c|c|c|c|c|c|c|c|}
\hline & \multicolumn{2}{|c|}{ Total $(n=65)$} & \multirow[b]{2}{*}{$P$} & \multicolumn{2}{|c|}{ Male $(n=31)$} & \multicolumn{2}{|c|}{ Female $(n=34)$} & \multirow[b]{2}{*}{$P$} \\
\hline & $\begin{array}{c}\text { MS(-) } \\
(n=50)\end{array}$ & $\begin{array}{c}\text { MS(+) } \\
(n=15)\end{array}$ & & $\begin{array}{c}\text { MS(-) } \\
(n=22)\end{array}$ & $\begin{array}{l}\text { MS }(+) \\
(n=9)\end{array}$ & $\begin{array}{c}\text { MS(-) } \\
(n=28)\end{array}$ & $\begin{array}{l}\text { MS }(+) \\
(n=6)\end{array}$ & \\
\hline $\mathrm{SV}(\mathrm{mL})$ & $63.5 \pm 9.8$ & $59.5 \pm 14.4$ & 0.065 & $66.7 \pm 10.2$ & $62.4 \pm 9.4$ & $61.8 \pm 19.8$ & $56.3 \pm 11.5$ & 0.767 \\
\hline $\mathrm{CO}(\mathrm{L})$ & $4.7 \pm 1.1$ & $4.2 \pm 0.9$ & 0.065 & $4.7 \pm 0.7$ & $4.3 \pm 1.0$ & $4.7 \pm 1.6$ & $4.2 \pm 0.8$ & 0.631 \\
\hline IVS (mm) & $1.0 \pm 0.7$ & $0.8 \pm 0.6$ & 0.167 & $0.9 \pm 0.1$ & $0.9 \pm 0.1$ & $1.0 \pm 1.3$ & $0.8 \pm 0.1$ & 0.265 \\
\hline PWT (mm) & $0.9 \pm 0.1$ & $0.9 \pm 0.1$ & 0.725 & $1.0 \pm 0.1$ & $1.0 \pm 0.1$ & $0.8 \pm 0.1$ & $0.8 \pm 0.1$ & 0.999 \\
\hline$E F(\%)$ & $61.3 \pm 6.0$ & $60.5 \pm 3.8$ & 0.465 & $59.5 \pm 7.0$ & $61.0 \pm 3.7$ & $62.8 \pm 5.5$ & $59.4 \pm 3.7$ & 0.595 \\
\hline FS (\%) & $32.8 \pm 4.5$ & $32.3 \pm 2.8$ & 0.74 & $32.3 \pm 3.5$ & $32.9 \pm 2.7$ & $33.9 \pm 4.8$ & $31.4 \pm 2.9$ & 0.104 \\
\hline LVM (g) & $176 \pm 36.7$ & $211.6 \pm 45.9$ & 0.012 & $205.9 \pm 44.5$ & $258.7 \pm 59.0$ & $141.3 \pm 21.0$ & $158.0 \pm 36.7$ & 0.001 \\
\hline LVMI $\left(\mathrm{g} / \mathrm{m}^{2}\right)$ & $1.8 \pm 0.7$ & $2.2 \pm 0.33$ & 0.065 & $1.9 \pm 0.4$ & $2.4 \pm 0.5$ & $1.6 \pm 0.2$ & $1.9 \pm 0.34$ & 0.053 \\
\hline MVE (m/sec) & $0.99 \pm 0.2$ & $0.93 \pm 0.3$ & 0.678 & $1.0 \pm 0.2$ & $0.9 \pm 0.2$ & $0.99 \pm 0.2$ & $0.95 \pm 0.2$ & 0.567 \\
\hline $\mathrm{MVA}(\mathrm{m} / \mathrm{sec})$ & $0.5 \pm 0.1$ & $0.5 \pm 0.1$ & 0.225 & $0.5 \pm 0.1$ & $0.5 \pm 0.1$ & $0.5 \pm 0.1$ & $0.5 \pm 0.1$ & 0.997 \\
\hline MVE/A & $1.9 \pm 0.6$ & $2.0 \pm 0.6$ & 0.559 & $1.8 \pm 0.4$ & $2.1 \pm 0.6$ & $2.0 \pm 0.5$ & $2.0 \pm 0.6$ & 0.273 \\
\hline MVE-DT (ms) & $152.6 \pm 25.7$ & $145.3 \pm 36.8$ & 0.381 & $159.7 \pm 28.8$ & $147.3 \pm 36.7$ & $148.3 \pm 21.8$ & $141.8 \pm 36.8$ & 0.886 \\
\hline EFT (PSAX) (mm) & $0.2 \pm 0.2$ & $0.2 \pm 0.04$ & 0.68 & $0.2 \pm 0.1$ & $0.2 \pm 0.03$ & $0.2 \pm 0.02$ & $0.2 \pm 0.04$ & 0.978 \\
\hline
\end{tabular}

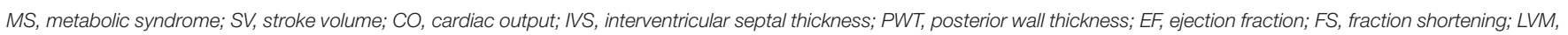

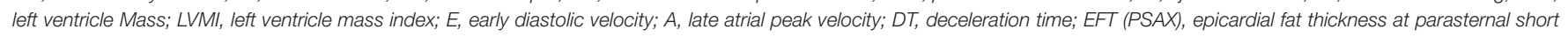
axis view.

prevalence of MS in obese children was $23.1 \%$ (15/65) similar to previous studies.

It seems appropriate to apply the same factors from adults in establishing a definition of MS in adolescent, such as the dyslipidemia, hypertension, and obesity which tend to track into adulthood (12). But some components need further correction in adolescents. For instance, impaired fasting glucose is very low but impaired glucose tolerance is fairly common in childhood $(13,14)$. So fasting glucose threshold need to be lowered and impaired glucose tolerance should be proposed as components of the MS (15). The cut off level for fasting glucose it our study is $110 \mathrm{mg} / \mathrm{dL}$, which is lower than that of ATPIII. Aside from this, 
TABLE 7 | Comparison of pulse wave velocity and ankle brachial index in obese adolescents.

\begin{tabular}{|c|c|c|c|c|c|c|}
\hline & \multicolumn{3}{|c|}{ Male $(n=31)$} & \multicolumn{3}{|c|}{ Female $(n=34)$} \\
\hline & $M s(-)(n=22)$ & $\operatorname{MS}(+)(n=9)$ & $P$ & $\operatorname{MS}(-)(n=28)$ & $\operatorname{MS}(+)(n=6)$ & $\boldsymbol{P}$ \\
\hline $\mathrm{HR}(/ \min )$ & $73.4 \pm 8.4$ & $70.6 \pm 9.2$ & 0.323 & $70.6 \pm 9.1$ & $75.2 \pm 10.1$ & 0.134 \\
\hline RbaPWV (cm/s) & $1102.8 \pm 120.1$ & $1117.8 \pm 138.2$ & 0.639 & $931.5 \pm 92.8$ & $946.5 \pm 100.4$ & 0.401 \\
\hline LbaPWV (cm/s) & $1130.2 \pm 115.1$ & $1112.0 \pm 143.2$ & 0.843 & $913.3 \pm 101.2$ & $952.1 \pm 103.2$ & 0.221 \\
\hline RABI & $102.8 \pm 7.9$ & $105.1 \pm 6.5$ & 0.321 & $105.8 \pm 7.2$ & $101.7 \pm 6.8$ & 0.088 \\
\hline LABI & $104.1 \pm 6.7$ & $106.7 \pm 5.4$ & 0.601 & $106.5 \pm 6.8$ & $100.4 \pm 7.9$ & 0.021 \\
\hline
\end{tabular}

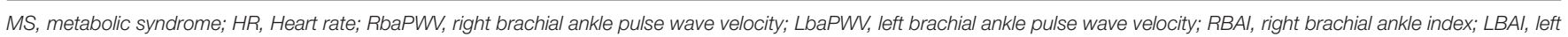
brachial ankle index.

TABLE 8 | Correlation between LV mass and other metabolic parameters.

\begin{tabular}{|c|c|c|c|c|c|}
\hline & Estimate & SE & Lower CL & Upper CL & $P$ \\
\hline BMI $\left(\mathrm{kg} / \mathrm{m}^{2}\right)$ & 4.385 & 1.244 & 1.883 & 6.886 & 0.009 \\
\hline $\mathrm{HC}(\mathrm{cm})$ & 2.121 & 0.639 & 0.835 & 3.406 & 0.017 \\
\hline Fat mass (kg) & 1.767 & 0.543 & 0.673 & 2.861 & 0.022 \\
\hline OI (\%) & 0.922 & 0.287 & 0.343 & 1.5 & 0.052 \\
\hline Insulin (mU/L) & 1.261 & 0.442 & 0.371 & 2.15 & 0.046 \\
\hline HOMA-IR & 5.06 & 1.841 & 1.359 & 8.762 & 0.084 \\
\hline TC (mg/dL) & 0.386 & 0.146 & 0.092 & 0.68 & 0.022 \\
\hline LDL-C (mg/dL) & 0.397 & 0.168 & 0.059 & 0.734 & 0.012 \\
\hline WC (cm) & 1.298 & 0.576 & 0.141 & 2.455 & 0.028 \\
\hline
\end{tabular}

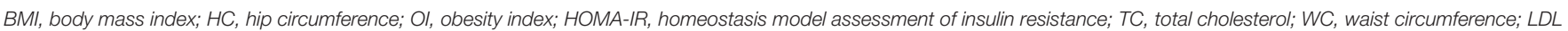
$-\mathrm{C}$, low density lipoprotein-cholesterol.

TABLE 9 | Correlations between visceral fat tissue and other metabolic parameters.

\begin{tabular}{|c|c|c|c|c|c|}
\hline Variable & Estimate & StdErr & LowerCL & UpperCL & $P$ \\
\hline WC (cm) & 1.074 & 0.19 & 0.692 & 1.455 & $<0.001$ \\
\hline DBP (mm Hg) & 0.476 & 0.205 & 0.065 & 0.887 & 0.024 \\
\hline $\mathrm{TG}(\mathrm{mg} / \mathrm{dL})$ & 0.118 & 0.041 & 0.036 & 0.199 & 0.006 \\
\hline LDL-C (mg/dL) & 0.203 & 0.065 & 0.072 & 0.335 & 0.003 \\
\hline TC (mg/dL) & 0.199 & 0.056 & 0.086 & 0.313 & 0.001 \\
\hline Glucose (mg/dL) & -0.59 & 0.285 & -1.163 & -0.018 & 0.044 \\
\hline BMI $\left(\mathrm{kg} / \mathrm{m}^{2}\right)$ & 2.537 & 0.43 & 1.673 & 3.401 & $<0.0001$ \\
\hline $\mathrm{HC}(\mathrm{cm})$ & 1.208 & 0.228 & 0.75 & 1.666 & $<0.0001$ \\
\hline WHR & 98.053 & 45.313 & 6.945 & 189.161 & 0.036 \\
\hline Ol & 0.553 & 0.101 & 0.35 & 0.756 & $<0.0001$ \\
\hline Fat \% & 1.743 & 0.388 & 0.962 & 2.524 & $<0.0001$ \\
\hline Fat mass (kg) & 1.138 & 0.179 & 0.777 & 1.498 & $<0.0001$ \\
\hline Insulin (mU/L) & 0.548 & 0.177 & 0.193 & 0.903 & 0.003 \\
\hline HOMA-IR & 1.958 & 0.75 & 0.451 & 3.466 & 0.012 \\
\hline Leptin (ug/L) & 0.792 & 0.381 & 0.024 & 1.56 & 0.043 \\
\hline
\end{tabular}

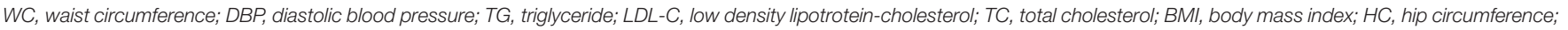
WHR, waist hip ratio; Ol, obesity index; HOMA-IR, homeostasis model assessment of insulin resistance.

the pubertal status, age range, ethnicity need to be considered in defining MS of adolescents $(1,7)$.

Visceral fat is usually measured by WC or other imaging methods. Among the parameters of MS, WC is easy and practical for screening children at risk of MS (2). Although WC is a better marker and widely used parameter of abdominal adipose tissue accumulation than the BMI, the increased WC is not sufficient to define visceral obesity alone, because of increased subcutaneous 
fat (16). In this study, we used abdominal sonographic method to estimate the visceral fat and found that VFT had significant correlation with WC.

The accumulation of visceral fat contrary to abdominal subcutaneous fat plays a significant role in the pathophysiology of the MS with insulin resistance in adolescent $(17,18)$. Visceral fat accumulation affects the adipose tissue to over secret leptin and hyposecretion of adiponectin (14).

Leptins correlate with BMI in children. High plasma leptin level is related to the development of essential hypertension, hyperinsulinemia and dyslipidemia (19). Adiponectin originates mainly from adipocytes and is an adipocytokine that is acting against atherogenic, diabetic and pro-inflammatory effect (8). Decreased adiponectin levels are associated with dysmetabolic state and cause MS. Furthermore, visceral obesity may lead to ectopic fat deposition in skeletal muscle, liver, heart, etc. (16). In this study, there was increased VFT in both MS male and MS female compared to control.

EFT at right ventricle, left ventricular apex and atrium seems to correlate with visceral fat and act as cardiometabolic risk factor (20). Measuring echocardiographic epicardial fat tissue can be used as a method for quantification of visceral fat. The epicardial adipose tissue contact and interact with the myocardial tissue and coronary arteries by secreting pro-inflammatory cytokines and vasoactive peptides (6). These molecules can develop glucose intolerance and atherosclerosis and increase cardiovascular risk $(6,21)$. In our study, we found an increased EFT both in MS males and MS females.

Obesity and MS demand increased metabolic and blood supply due to greater adipose tissue, which increase blood volume and cardiac preload. And vascular alterations such as arterial stiffness and peripheral resistance increase afterload to the heart. Finally, they incur LV hypertrophy and LV diastolic dysfunction $(22,23)$. In our study, there was significant increase in LVM and LVMI in MS male. MS female also showed some trend of increased LVM without significance. In short, this study found VFT and LVM is significantly correlated with metabolic and cardiac parameters. So, screening for the MS in overweight adolescents may help to predict future cardiovascular disease.

As CVD is mainly result of atherosclerosis, the predictors of atherosclerosis are important. And CIMT is a highly predictive marker of progression of atherosclerosis in patients with MS (24). However, we could not find abnormal CIMT from both MS and non-MS groups in this study. This might be due to young age groups whose peripheral artery is very resistant to local vascular inflammation and atherosclerosis progression. Also, baPWV data from our study was not efficient either. In our study, female LABI only showed statistically significant difference. The reason why there was no statistical difference between the remaining data is believed to be due to the low accuracy of the test. Because baPWV method include peripheral vessels, it is less accurate and does not reflect the degree of arteriosclerosis in the central blood vessels, but rather changes in peripheral arteries can be reflected. Carotid-femoral PWV (cfPWV) is better alternative since it predicted cardiovascular mortality (25). However, femoral artery waveforms in cfPWV measure are difficult to accurately record in metabolic syndrome, obesity, diabetes, peripheral artery patients. So, we suggest other non-invasive and high accuracy central blood pressure measurements such as radial tonometry or brachial cuff pulse volume plethysmography for future research (26).

Previous studies of MS in adolescents showed abundant evidence of risk factors for CVD (18). For example, autopsy studies in childhood showed that obesity, high BP, high TG, and low HDL-C are related to coronary atherosclerosis $(27,28)$. So, early identification of these risk factors is important in chronic disease prevention $(29,30)$. In the present study, we found significant differences in VFT and LVM in obese adolescents with MS.

Hyperinsulinemia and insulin resistance are well-known risk factors for CVD. Hyperinsulinemia directly contributes to deteriorated renal function and increased risk of CVD (31). The foremost mechanism is an up-regulation of the renin-angiotensin system, which contribute to begin hypertension, heart failure and atherosclerosis (32). These hyperinsuliemia, insulin resistance and finally upregulated renin-angiotensin system, are thought to contribute to endothelial dysfunction, atherosclerosis and CVD (32). Another story of insulin dysregulation is insulin resistance syndrome (IRS). IRS comprise of hyperinsulinemia, dyslipidemia, hypertension, and obesity. A child whose parents have IRS have greater insulin resistance and CVD risks compared with those who do not (33). So, IRS in adolescent is of potential importance, because of the high prevalence of impaired glucose tolerance and obesity, and the increasing incidence of type 2 diabetes in adolescent (34).

Adolescent obesity-induced other metabolic complication is hyperuricemia. In our study, we did not collect uric acid levels. But uric acid is associated with cardiovascular, renal and metabolic diseases. It is because serum uric acid (SUA) is known as an antioxidant factor in the extracellular space, but as a pro-oxidant factor inside the cell (35).

Among these diseases, the link between SUA levels and hypertension seem to be the strongest. When children with high SUA were followed for 10 years, high incidence of MS in male subjects are observed (36). Also increased SUA is associated with new-onset primary hypertension in children (37). It is noted that hypertensive children with elevated SUA have a higher prevalence of obesity-related CVD (38). And one observational cohort study shows uric acid elevation is commonly found in hypertriglyceridemia, obesity, insulin resistance and hypertension. But they failed to show low uric acid level in well-controlled hypertension patients. Until now, whether elevated uric acid level actually causes CVD or increase MS remains to be confirmed (39).

There are several limitations to this study. First, the study included small cohort not enough to analyze all the data for each gender. Further research with large adolescent population is inevitable. Second, there is absence of follow up data. Third, there is lack of comprehensive assessment according to diverse adolescent age group, because the age range is confined to 15-18. Finally, even though measurements were conducted by same sonographer, there could be some intraobserver variability of reproducibility of measuring the parameters such as IMT, VFT, and EFT due to the sonographer's subjectivity. This could have been avoided by performing three measurements and averaging them. 
Since the time between adolescent obesity and occurrence of clinical disease is relatively short, longitudinal prospective design dealing from adolescence to adulthood is needed to explore the complexity of interrelation of MS risk factors. The prospective study of longer time course may offer a better opportunity in adolescent obesity and CVD risk research.

Overall, We defined adolescent MS as to meet more than 3 categories (WC $\geq 90$ th percentile, $\mathrm{BP} \geq 90$ th percentile, $\mathrm{TG} \geq$ $110 \mathrm{mg} / \mathrm{dL}, \mathrm{HDL} \leq 40 \mathrm{mg} / \mathrm{dL}$, fasting glucose $\geq 110 \mathrm{mg} / \mathrm{dL}$ ). The study subjects are obese male and female adolescents defined as BMI above the 95th percentile for age and sex by the survey of Korean Centers of Disease Control and Prevention in 2007. In this setting, we found both VFT in inn total and female MS group and LVM in total and male adolescents were increased. Although there were statistical differences between male and female adolescents, it is meaningful that there were differences in VFT and LVM between the entire MS and non-MS groups.

In conclusion, one can assume that VFT and LVM are appropriate and possible markers for predicting increased future cardiovascular risk in obese adolescents.

\section{REFERENCES}

1. Ford ES, Li C. Defining the metabolic syndrome in children and adolescents: will the real definition please stand up? J Pediatr. (2008) 152:1604. doi: 10.1016/j.jpeds.2007.07.056

2. Zimmet P, Alberti KG, Kaufman F, Tajima N, Silink M, Arslanian S, et al. The metabolic syndrome in children and adolescents - an IDF consensus report. Pediatr Diabetes. (2007) 8:299-306. doi: 10.1111/j.1399-5448.2007.00271.x

3. McCracken E, Monaghan M, Sreenivasan S. Pathophysiology of the metabolic syndrome. Clin Dermatol. (2018) 36:1420. doi: 10.1016/j.clindermatol.2017.09.004

4. Neeland IJ, Ross R, Després JP, Matsuzawa Y, Yamashita S, Shai $\mathrm{I}$, et al. International atherosclerosis society; international chair on cardiometabolic risk working group on visceral obesity. Visceral and ectopic fat, atherosclerosis, and cardiometabolic disease: a position statement. Lancet Diabetes Endocrinol. (2019) 7:715-25. doi: 10.1016/S2213-8587(19)30084-1

5. Spoto B, Betta ED, Mattace-Raso F, Sijbrands E, Vilardi A, Parlongo RM, et al. Pro- and anti-inflammatory cytokine gene expression in subcutaneous and visceral fat in severe obesity. Nutr Metab Cardiovasc Dis. (2014) 24:113743. doi: 10.1016/j.numecd.2014.04.017

6. Iacobellis G, Barbaro G. The double role of epicardial adipose tissue as pro- and anti-inflammatory organ. Horm Metab Res. (2008) 40:4425. doi: $10.1055 / \mathrm{s}-2008-1062724$

7. Korner A, Kratzsch J, Gausche R, Schaab M, Erbs S, Kiess W. New predictors of the metabolic syndrome in children-role of adipocytokines. Pediatr Res. (2007) 6:640-5. doi: 10.1203/01.pdr.0000262638.48304.ef

8. Gnacińska M, Małgorzewicz S, Stojek M, Łysiak-Szydłowska W, Sworczak K. Role of adipokines in complications related to obesity: a review. Adv Med Sci. (2009) 54:150-7. doi: 10.2478/v10039-009-0035-2

9. Chen W, Srinivasan SR, Elkasabany A, Berenson GS. Cardiovascular risk factors clustering features of insulin resistance syndrome (Syndrome $\mathrm{X}$ ) in a biracial (Black-White) population of children, adolescents, and young adults: the Bogalusa Heart Study. Am J Epidemiol. (1999) 150:66774. doi: 10.1093/oxfordjournals.aje.a010069

10. Oh KW, Jang MJ, Lee NY, Moon JS, Lee CG, Yoo MH, et al. Prevalence and trends in obesity among Korean children and adolescents in 1997 and 2005. Korean J Pediatr. (2008) 51:950-5. doi: 10.3345/kjp.2008.51.9.950

11. Syed-Abdul MM, Soni DS, Barnes JT, Wagganer JD. Comparative analysis of BIA, IBC and DXA for determining body fat in American Football players. J Sports Med Phys Fitness. (2021) 61:687-92. doi: 10.23736/S0022-4707.21.11278-2

\section{DATA AVAILABILITY STATEMENT}

The raw data supporting the conclusions of this article will be made available by the authors, without undue reservation.

\section{ETHICS STATEMENT}

The studies involving human participants were reviewed and approved by IRB at Ewha Womans University (Approval No: 0319/12/06.2020). Written informed consent to participate in this study was provided by the participants' legal guardian/next of kin.

\section{AUTHOR CONTRIBUTIONS}

JL, YH, and HK: conceptualization. JL: methodology, formal analysis, data curation, writing-original draft, and supervision. HK: investigation. YH and HK: writing-review and editing. All authors contributed to the article and approved the submitted version.

12. Cook S, Weitzman M, Auinger P, Nguyen M, Dietz WH. Prevalence of a metabolic syndrome phenotype in adolescents: find-ings from the third National Health and Nutrition Examination Survey, 1988-1994. Arch Pediatr Adolesc Med. (2003) 157:821-7. doi: 10.1001/archpedi.157. 8.821

13. Sinha R, Fisch G, Teague B, Tamborlane WV, Banyas B, Allen K, et al. Prevalence of impaired glucose tolerance among children and adolescents with marked obesity. $N$ Engl J Med. (2002) 346:802-10. doi: 10.1056/NEJMoa012578

14. Goran MI, Bergman RN, Avila Q, Watkins M, Ball GD, Shaibi GQ, et al. Impaired glucose tolerance and reduced beta-cell function in overweight Latino children with a positive family history for type 2 diabetes. J Clin Endocrinol Metab. (2004) 89:207-12. doi: 10.1210/jc.2003-031402

15. The Expert Committee on the Diagnosis and Classification of Diabetes Mellitus. Follow-up report on the diagnosis of diabetes mellitus. Diabetes Care. (2003) 26:3160-7. doi: 10.2337/diacare.26.11.3160

16. Després JP, Lemieux I, Bergeron J, Pibarot P, Mathieu P, Larose E, et al. Abdominal obesity and the metabolic syndrome: contribution to global cardiometabolic risk. Arterioscler Thromb Vasc Biol. (2008) 28:103949. doi: 10.1161/ATVBAHA.107.159228

17. Steinberger J, Daniels SR, Eckel RH, Hayman L, Lustig RH, McCrindle B, et al. American Heart Association atherosclerosis, hypertension, and obesity in the Young Committee of the Council on Cardiovascular Disease in the Young; Council on Cardiovascular Nursing; and Council on Nutrition, Physical Activity, and Metabolism. Progress and challenges in metabolic syndrome in children and adolescents: a scientific statement from the American Heart Association Atherosclerosis, Hypertension, and Obesity in the Young Committee of the Council on Cardiovascular Disease in the Young; Council on Cardiovascular Nursing; and Council on Nutrition, Physical Activity, and Metabolism. Circulation. (2009) 119:62847. doi: 10.1161/CIRCULATIONAHA.108.191394

18. Cruz ML, Goran MI. The metabolic syndrome in children and adolescents. Curr Diabetes Rep. (2004) 4:53-62. doi: 10.1007/s11892-004-0012-x

19. Lago F, Gómez R, Gómez-Reino JJ, Dieguez C, Gualillo O. Adipokines as novel modulators of lipid metabolism. Trends Biochem Sci. (2009) 34:50010. doi: 10.1016/j.tibs.2009.06.008

20. Gastaldeli A, Basta G. Ectopic fat and cardiovascular disease: what is the link? Nutr Metab Cardiovasc Dis. (2010) 20:48190. doi: 10.1016/j.numecd.2010.05.005

21. Iacobellis G, Gao YJ, Sharma AM. Do cardiac and perivascular adipose tissue play a role in atherosclerosis? Curr Diab Rep. (2008) 8:20-4. 
22. Bokor S, Frelut ML, Vania A, Hadjiathanasiou CG, Anastasakou M, Malecka-Tendera E, et al. Prevalence of metabolic syndrome in European children. Int J Pediatr Obes. (2008) 2:3-8. doi: 10.1080/174771608024 04509

23. Cote AT, Harris KC, Panagiotopoulos C, Sandor GG, Devlin AM. Childhood obesity and cardiovascular dysfunction. J Am Coll Cardiol. (2013) 62:130919. doi: 10.1016/j.jacc.2013.07.042

24. Mazurek T, Zhang L, Zalewski A, Mannion JD, Diehl JT, Arafat H, et al. Human epicardial adipose tissue is a source of inflammatory mediators. Circulation. (2003) 108:2460-6. doi: 10.1161/01.CIR.0000099542.57313.C5

25. Pannier B, Guérin AP, Marchais SJ, Safar ME, London GM. Stiffness of capacitive and conduit arteries. Prognostic significance for end-stage renal disease patients. Hypertension. (2005) 45:592-6. doi: 10.1161/01.HYP.0000159190.71253.c3

26. McEniery CM, Cockcroft JR, Roman MJ, Franklin SS, Wilkinson IB. Central blood pressure: current evidence and clinical importance. Eur Heart J. (2014) 35:1719-25. doi: 10.1093/eurheartj/eht565

27. Berenson GS, Srinivasan SR, Bao W, Newman WP, Tracy RE, Wattigney WA. Association between multiple cardiovascular risk factors and atherosclerosis in children and young adults. The Bogalusa Heart Study. N Engl J Med. (1998) 338:1650-56. doi: 10.1056/NEJM199806043382302

28. McGill HC, McMahan CA, Herderick EE, Zieske AW, Malcom GT, Tracy RE, et al. Pathobiological determinants of atherosclerosis in youth (PDAY) research group. Obesity accelerates the progression of coronary atherosclerosis in young men. Circulation. (2002) 105:27128. doi: 10.1161/01.CIR.0000018121.67607.CE

29. Sardinha LB, Santos DA, Silva AM, Grøntved A, Andersen LB, Ekelund U. A comparison between BMI, waist circumference, and waist-toheight ratio for identifying cardio-metabolic risk in children and adolescents. PLOS ONE. (2016) 11:e0149351. doi: 10.1371/journal.pone. 0149351

30. Wicklow BA, Becker A, Chateau D, Palmer K, Kzyrskij A, Sellers EA. Comparison of anthropometric measurements in children to predict metabolic syndrome in adolescence: analysis of prospective cohort data. Int J Obes. (2015) 39:1070-8. doi: 10.1038/ijo.2015.55

31. Chen J, Muntner P, Hamm LL, Fonseca V, Bautman V, Whelton $\mathrm{PK}$, et al. Insulin resistance and risk of chronic kidney disease in nondiabetic US adults. J Am Soc Nephrol. (2003) 14:469-77. doi: 10.1097/01.ASN.0000046029.5333.09

32. Liu Z. The renin-angiotensin system and insulin resistance. Curr Diab Rep. (2007) 7:34-42. doi: 10.1007/s11892-007-0007-5
33. James SP, David RJ, Julia S, Antoinette M, Alan RS. Insulin resistance and cardiovascular disease risk factors in children of parents with the insulin resistance (metabolic) syndrome. Diabetes Care. (2004) 27:77580. doi: 10.2337/diacare.27.3.775

34. Rosenbloom AL, Joe JR, Young RS, Winter WE. Emerging epidemic of type 2diabetes in youth. Diabetes Care. (1999) 22:345-54. doi: 10.2337/diacare.22.2.345

35. Johnson RJ, Sánchez-Lozada LG, Mazzali M, Feig DI, Kanbay M, Sautin YY. What are the key arguments against uric acid as a true risk factor for hypertension? Hypertension. (2013) 61:948-51. doi: 10.1161/HYPERTENSIONAHA.111.00650

36. Sun HL, Pei D, Lue KH, Chen YL. Uric acid levels can predict metabolic syndrome and hypertension in adolescents: a 10-year longitudinal study. PLoS ONE. (2015) 10:e0143786. doi: 10.1371/journal.pone.0143786

37. Feig DI, Johnson RJ. Hyperuricemia in childhood primary hypertension. Hypertension. (2003) 42:247-52. doi: 10.1161/01.HYP.0000085858.66548.59

38. Lauren DR, Edgar RM, Jeffrey JF, Lauren FL, Kathryn WH, Lawrence JA, et al. Elevated uric acid and obesity-related cardiovascular disease risk factors among hypertensive youth. Pediatric Nephrology. (2015) 30:216976. doi: 10.1007/s00467-015-3154-y

39. Michael HA, Hillel C, Shantha M, Salah K. Serum Uric Acid and Cardiovascular Events in Successfully Treated Hypertensive Patients. Hypertension. (1999) 34:144-50. doi: 10.1161/01.HYP.34.1.144

Conflict of Interest: The authors declare that the research was conducted in the absence of any commercial or financial relationships that could be construed as a potential conflict of interest.

Publisher's Note: All claims expressed in this article are solely those of the authors and do not necessarily represent those of their affiliated organizations, or those of the publisher, the editors and the reviewers. Any product that may be evaluated in this article, or claim that may be made by its manufacturer, is not guaranteed or endorsed by the publisher.

Copyright (c) 2021 Lee, Hong and Kim. This is an open-access article distributed under the terms of the Creative Commons Attribution License (CC BY). The use, distribution or reproduction in other forums is permitted, provided the original author(s) and the copyright owner(s) are credited and that the original publication in this journal is cited, in accordance with accepted academic practice. No use, distribution or reproduction is permitted which does not comply with these terms. 